\title{
Interleukin-1 receptor antagonist-mediated neuroprotection by umbilical cord-derived mesenchymal stromal cells following transplantation into a rodent stroke model
}

\author{
Seung-Hun Oh', Chunggab Choi ${ }^{2}$, Jeong-Eun Noh², Nayeon Lee², Yong-Woo Jeong ${ }^{2}$, Iksoo Jeon², Jeong-Min Shin ${ }^{3,4}$,
} Ji-Hye Kim³ ${ }^{3}$ Ho-Jin Kim³ ${ }^{3}$ Ji-Min Lee ${ }^{1}$, Hyun-Sook Kim¹, Ok-Joon Kim ${ }^{1}$ and Jihwan Song ${ }^{2,5}$

\begin{abstract}
The human umbilical cord is a promising source of mesenchymal stromal cells (MSCs). Intravenous administration of human umbilical cord-derived MSCs (IV-hUMSCs) showed a favorable effect in a rodent stroke model by a paracrine mechanism. However, its underlying therapeutic mechanisms must be determined for clinical application. We investigated the therapeutic effects and mechanisms of our good manufacturing practice (GMP)-manufactured hUMSCs using various cell doses and delivery time points in a rodent model of stroke. IV-hUMSCs at a dose of $1 \times 10^{6}$ cells at $24 \mathrm{~h}$ after stroke improved functional deficits and reduced neuronal damage by attenuation of post-ischemic inflammation. Transcriptome and immunohistochemical analyses showed that interleukin-1 receptor antagonist (IL-1ra) was highly upregulated in ED-1-positive inflammatory cells in rats treated with IV-hUMSCs. Treatment with conditioned medium of hUMSCs increased the expression of IL-1ra in a macrophage cell line via activation of cAMPresponse element-binding protein (CREB). These results strongly suggest that the attenuation of neuroinflammation mediated by endogenous IL-1ra is an important therapeutic mechanism of IV-hUMSCs for the treatment of stroke.
\end{abstract}

\section{Introduction}

Neuroprotection and tissue repair in the injured brain following cerebral ischemia are important targets to develop a successful stroke therapy. Cell therapy using mesenchymal stromal cells (MSCs) has been regarded as a potent approach to treat stroke ${ }^{1,2}$. There is numerous experimental evidence showing that intravenous administration of MSCs induces functional improvement in

Correspondence: Ok-Joon Kim(okjun77@cha.ac.kr) or Jihwan Song (jsong@cha. ac.kr)

'Department of Neurology, CHA Bundang Medical Center, CHA University, Seongnam, Republic of Korea

2Department of Biomedical Science, CHA University, Seongnam, Republic of Korea

Full list of author information is available at the end of the article

These authors contributed equally: Seung-Hun Oh, Chunggab Choi, Jeong-Eun Noh. cerebral ischemia through paracrine or endocrine signaling to the target tissues. MSCs secrete multiple trophic factors, including vascular endothelial growth factor (VEGF) and hepatocyte growth factor (HGF), which promote tissue repair in the damaged brain $^{3}$. In addition, MSCs have strong immune-modulating properties. Under specific circumstances, MSCs not only reduce the activation of pro-inflammatory cytokines (i.e., interleukin (IL)-1 and tumor necrosis factor $\alpha(\mathrm{TNF}-\alpha)$ ) but also enhance the expression of anti-inflammatory cytokines (i.e., transforming growth factor $\beta$ (TGF- $\beta$ ), IL-10, and indoleamine 2, 3-dioxygenase (IDO)) in immune cells ${ }^{3}$. These strong regenerative and immune-modulating properties of MSCs can provide multi-modal therapeutic functions in various diseases, including stroke. The human umbilical cord contains numerous populations of MSC-like cells ${ }^{4}$. Previous studies have shown that

\section{(c) The Author(s) 2018}

\footnotetext{
(c) (1) (-) Open Access This article is licensed under a Creative Commons Attribution-NonCommercial-NoDerivatives 4.0 International License, which permits any non-commercial cc) ${ }_{\text {BY NC ND }}$ use, sharing, distribution and reproduction in any medium or format, as long as you give appropriate credit to the original author(s) and the source, and provide a link to the Creative Commons license. You do not have permission under this license to share adapted material derived from this article or parts of it. The images or other third party material in this article are included in the article's Creative Commons license, unless indicated otherwise in a credit line to the material. If material is not included in the article's Creative Commons license and your intended use is not permitted by statutory regulation or exceeds the permitted use, you will need to obtain permission directly from the copyright holder. To view a copy of this license http:// creativecommons.org/licenses/by-nc-nd/4.0/
} 
intraparenchymal transplantation or intravenous administration of human umbilical cord-derived MSCs (hUMSCs) improves functional recovery in animal models of stroke ${ }^{5,}{ }^{6}$, indicating that hUMSCs can be a potent source for cell therapy in stroke. However, many unresolved issues must be addressed before clinical application of hUMSCs to treat human stroke. In particular, related preclinical data to explain the therapeutic mechanism of intravenous administration of hUMSCs (IV-hUMSCs) to treat stroke are still largely lacking. Here, we performed a comprehensive preclinical experiment to determine the effect of good manufacturing practice (GMP)-manufactured hUMSCs and investigated their therapeutic mechanisms in a rodent model of stroke.

\section{Materials and methods}

\section{Ethics statements}

This study was approved by the Institutional Review Board at the CHA Bundang Medical Center for the use of umbilical cord (IRB no.: BD2013-004D). All experimental animals were manipulated in accordance with guidelines provided by the Institutional Animal Care and Use Committee of CHA University (IACUC no.: 090012).

\section{Preparation of hUMSCs}

With informed consent from a single healthy donor, cells were retrieved from the umbilical cord at CHA Bundang Medical Center (Seongnam, Republic of Korea) and prepared immediately. Preparations of hUMSCs were conducted in the GMP facility, and the isolation and expansion of hUMSCs were performed according to the Good Clinical Practice (GCP) guidelines of the Master Cell Bank. To isolate hUMSCs, we sliced Wharton's jelly into $1-5-\mathrm{mm}$ explants after the umbilical vessels were removed. Isolated slices were attached to $\alpha$-MEM (HyClone, IL) supplemented with 10\% FBS (HyClone, IL), FGF4 (R\&D Systems, MN), and heparin (SigmaAldrich, MO) on culture plates and subsequently cultured. The medium was changed every 3 days. After 15 days, the umbilical cord fragments were discarded, and the cells were passaged with TrypLE (Invitrogen, MA) and expanded until they reached sub-confluence (80-90\%). The cells were incubated under hypoxic conditions $(3 \%$ $\mathrm{O}_{2}, 5 \% \mathrm{CO}_{2}$, and $37^{\circ} \mathrm{C}$ ). The hUMSCs at passage 7 were used in the present study. Karyotype analysis confirmed that the cells contained a normal human karyotype. Using reverse transcriptase PCR, the absence of viral pathogens (human immunodeficiency virus-1 and 2, cytomegalovirus, hepatitis $\mathrm{B}$ virus, hepatitis $\mathrm{C}$ virus, human $\mathrm{T}$ lymphocytic virus, Epstein-Barr virus, and mycoplasma) in cell pellets was confirmed. To identify the immunophenotype of hUMSCs, fluorescence-activated cell sorting (FACS) analysis was performed as previously described ${ }^{7}$.
The hUMSCs expressed high levels of cell surface markers for MSCs (CD44, CD73, CD90, and CD105), but the expression of markers for hematopoietic stem cells (CD31, CD34, and CD 45) and HLA-DR was negligible (Supplementary Figure S1a). The cells could be efficiently differentiated into adipocytes, osteocytes, and chondrocytes (Supplementary Figure S1b).

When hUMSCs $(n=3)$ reached $100 \%$ confluence, they were further cultured in serum-free medium for $48 \mathrm{~h}$, and the hUMSCs-CM was collected. Subsequently, the protein concentration of TGF- $\beta 1$ (Human TGF- $\beta 1$ ELISA kit, R\&D Systems, MN), VEGF (Human VEGF ELISA kit, R\&D Systems, MN), HGF (Human HGF ELISA kit, Cloud-Clone Corp., TX), and IDO (Human IDO ELISA kit, BlueGene Biotech., Shanghai, China) from the hUMSCs-CM was measured using commercially available enzyme-linked immunosorbent assay (ELISA) kits according to the manufacturer's instructions. The hUMSCs secreted high levels of IDO, TGF- $\beta 1$, and HGF (Supplementary Figure S1c). These data indicate that hUMSCs have characteristics of MSCs and secrete cytokines and trophic factors that are involved in the immune response and tissue repair ${ }^{3,8}$.

\section{Rodent stroke model and intravenous administration of hUMSCs}

A total of 151 male Sprague-Dawley rats weighing 270-300 g were used for a 90-min MCAo method developed by Longa et $\mathrm{al}^{9}$. The detailed method of MCAo induction has been described previously ${ }^{10}$. For the doseand delivery time-response study of IV-hUMSCs, the MCAo-induced rats were randomly divided into five groups ( $n=10$ per group) as follows: group 1 (G1) included rats treated with saline at $24 \mathrm{~h}$ post MCAo; group 2 (G2), $1 \times 10^{5} \mathrm{IV}$-hUMSCs at $24 \mathrm{~h}$ post MCAo; group 3 (G3), $5 \times 10^{5} \mathrm{IV}$-hUMSCs at $24 \mathrm{~h}$ post MCAo; group 4 (G4), $1 \times 10^{6} \mathrm{IV}$-hUMSCs at $24 \mathrm{~h}$ post MCAo; group 5 (G5), $1 \times 10^{6} \mathrm{IV}$-hUMSCs at 7 days post MCAo. Cells mixed with $500 \mu \mathrm{l}$ of saline were carefully infused into tail veins of the corresponding treatment group for 5 $\mathrm{min}$ at the appropriate delivery time points. No profound bleeding occurred during treatment, and vital signs in all rats were stable during the procedure. All rats were injected with cyclosporine A $(5 \mathrm{mg} / \mathrm{kg})$ intraperitoneally from the previous day of cell infusion and up to 8 weeks of cell administration. After completion of the dose- and time-response studies, another independent experiment was performed to confirm the effects of treatment with $1 \times 10^{6}$ IV-hUMSCs at $24 \mathrm{~h}$ post MCAo. During 4 weeks after cerebral ischemia, functional scores were compared between rats treated with $1 \times 10^{6}$ IV-hUMSCs at $24 \mathrm{~h}$ after MCAo (IV-hUMSC group, $n=10$ ) and rats treated with saline (saline group, $n=10$ ). 


\section{Behavioral tests}

Behavioral tests were conducted by independent investigators who were blinded to treatment group. The rotarod test and mNSS test were performed as previously described $^{11}$. For the rotarod test, each rat was pre-trained three times a day for 3 consecutive days before MCAo induction to reduce variation among animals. The rat was placed on the rotarod wheel to record the time of endurance on the wheel. The rod speed of the rotarod device was gradually increased from 4 to 40 r.p.m. for 2 min. We recorded the time that it took for each rat to fall down from the rotating wheel, and calculated the average time from three trials. The test was conducted 1 day before MCAo (pre), on the day (D0) and 2 days after MCAo (D2). Afterward, the test was conducted once per week up to 8 weeks. For the mNSS test, each rat was tested 1 day after MCAo induction and weekly up to 8 weeks after cell administration. The rat was given a score, which was the sum of the individual neurological test scores. A high score represents the most severe condition, whereas a low score represents the normal condition.

\section{Measurement of the infarct size}

At 8 weeks post MCAo, cresyl violet staining was used to measure the infarct volume in the MCAo models $(n=$ 7 for each group). In an independent in vivo experiment group, the infarct size was compared at $72 \mathrm{~h}$ post MCAo (48 h after treatment) using 2, 3, 5-triphenyltetrazolium chloride (TTC) staining between the IV-hUMSC and saline treatment ( $n=5$ for each group). The detailed tissue preparation methods have been described previously $^{11,12}$. We estimated the infarct size as a percentage of the intact contralateral hemisphere by use of the following equation: estimated infarct size $(\%)=[1-($ area of remaining ipsilateral hemisphere/area of intact contralateral hemisphere) $] \times 100$. The areas of interest were measured with ImageJ software (ImageJ, National Institutes of Health), and the values were summed for six serial coronal sections per brain.

\section{Immunohistochemistry}

To investigate the effect of treatment with IV-hUMSCs on the pathological changes in the ischemic brain, immunohistochemical analyses were conducted at $72 \mathrm{~h}$ after MCAo induction ( $n=5$ for each group). Detailed methodology for tissue preparation for immunohistochemistry has been described elsewhere ${ }^{10,}{ }^{11}$. Immunohistochemical markers, such as those for macrophages/ microglia (ED-1, Iba-1, iNOS, and CD206), neutrophils (ELANE), rat endothelial cells (Reca-1), human nucleus $(\mathrm{hNu})$, and interleukin-1 receptor antagonist (IL-1ra), were used to evaluate the changes in the ischemic brain after IV-hUMSC treatment. Detailed information for individual antibodies used for immunohistochemistry is provided elsewhere (Supplementary Table S1). The TUNEL assay for apoptosis was performed as previously described ${ }^{10}$. The sections were counterstained with the nuclear marker 4', 6-diamidine-29-phenylindole dihydrochloride (DAPI). Fluorescently labeled specimens were viewed under a confocal laser-scanning microscope (LSM510; Carl Zeiss Microimaging Inc., München, Germany).

\section{Whole gene expression analysis of brain tissues}

At $72 \mathrm{~h}$ post MCAo, the ipsilateral hemisphere subjected to MCAo was used for mRNA microarray analysis. RNA was isolated as quickly as possible from the ipsilateral hemisphere to MCAo in rats without MCAo (control group, $n=5$ ), MCAo rats treated with $1 \times 10^{6} \mathrm{IV}$ hUMSCs $(n=6)$, and MCAo rats treated with saline $(n=$ 5) at $24 \mathrm{~h}$ post MCAo by homogenization with $\mathrm{TRIzol}^{\oplus}$ reagent (Thermo Fisher Scientific, MA) and RNeasy columns (Qiagen, Hilden, Germany). In addition to the sham control (i.e., saline-treated group), we also used normal rat brains without MCAo induction as an additional control to investigate the changes in inflammatory gene expression after treatment with IV-hUMSCs in cerebral ischemia, To ensure RNA quality, only samples with an optical density (OD) of $260 \mathrm{~nm} / 280 \mathrm{~nm}$ ratio above 1.8 with an Agilent 2100 Bioanalyzer (Agilent Technologies, CA) were used for microarray analysis. RNA labeling and purification were performed, and the samples were hybridized to Agilent rat mRNA microarray chips (SurePrint G3 Rat Gene Expression $8 \times 60$ k, Agilent Inc., CA) according to the manufacturer's instructions. The array was scanned using the Agilent Technologies G2600DSG12494263 (Agilent Inc., CA). Array data exporting processing and analysis were performed using Agilent Feature Extraction software (v100.0.1.1). The data were filtered by log transformation and quantile normalization. The array data were statistically analyzed using the Student's $t$ test with false discovery rate correction (Benjamini-Hochberg test) for pairwise comparisons among each group. A differentially expressed transcript was described as a gene with a more than twofold difference (FD) and significant difference in the corrected $p$ value $(p)<0.01$ for consideration of the multiplecomparison hypothesis. All data analyses and visualization of differentially expressed transcripts were conducted using R 3.0.1 (www.r-project.org). The microarray data are registered in the GEO repository (accession no. GSE78731).

\section{Quantitative real-time PCR of rat brain tissues}

Real-time PCR was performed using the same tissue samples that were used in the microarray test to investigate inflammatory cytokines that are known to be related 
to the stroke pathophysiology ${ }^{13}$, including $I L 1 R N, I L 1 B$, TNF, IL6, MMP9, IL4, IL10, and TGFB1. In this study, we also used RNA from normal rat brains as well as salinetreated MCAo rat brains as controls to investigate the changes in inflammatory gene expression after treatment with IV-hUMSCs in cerebral ischemia. Primer sets for each gene are described in Supplementary Table S2. Total RNAs were reverse-transcribed to the complementary DNA strand using the SuperScript ${ }^{\circledast}$ II First-Strand Synthesis System (Invitrogen, MA). Expression of mRNAs was quantified using the CFX ${ }^{\mathrm{TM}}$ real-time system (Bio-Rad Laboratories, CA) and Quantitect ${ }^{\circledR}$ SYBR Green PCR kit (Qiagen, Hilden, Germany). The real-time PCR was duplicated for each gene, and the mean value was used for the statistical analysis. The mRNA levels of selected genes were normalized to GAPDH. The fold difference is represented as a $2^{- \text {ddCT }}$ value that was calculated by the comparative threshold $\left(\mathrm{C}_{\mathrm{T}}\right)$ cycle method.

\section{Treatment of raw 264.7 cells with the conditioned medium of hUMSCs}

A mouse macrophage cell line (raw 264.7 cells, ATCC, VA) was cultured according to the manufacturer's instructions. The raw 264.7 cells were treated with either LPS (100 ng/ml, Sigma-Aldrich, MO) or LPS together with hUMSCs-CM for $24 \mathrm{~h}$, and the supernatants were isolated from each treated group.

\section{Western blot analysis}

After homogenization of raw 264.7 cells, proteins were isolated using a protein lysis buffer (PRO-PREP ${ }^{\mathrm{mx}}$, Intron Biotechnology, Seongnam, Republic of Korea) and subjected to immunoblot analysis according to the manufacturer's instructions. Whole proteins were separated on SDS-PAGE gels and immunoblotted using primary antibodies. The primary antibodies used are as follows: (1) anti-CREB and anti-p-CREB (1:1000, Cell Signaling Technology, MA); (2) anti-NF- $\mathrm{BB}$ p65 and anti-p-NF-кB p65 (1:1000, Santa Cruz Biotechnology, TX), and (3) antiIL-1ra (1:500, Santa Cruz Biotechnology, TX). GAPDH (1:5000, Santa Cruz Biotechnology, TX) was used as an internal control. Quantification of the bands was performed using the NIH ImageJ program. Independent experiments were performed in triplicate on different days.

\section{Inhibition of p-CREB}

After the raw 264.7 cells $\left(2 \times 10^{5}\right)$ were seeded onto the plate and incubated for $24 \mathrm{~h}$, they were pretreated with serum-free medium containing KG501 (2, 5, and $10 \mu \mathrm{M}$, Sigma-Aldrich, MO) for $45 \mathrm{~min}$. The supernatant was then removed from the dish, and the cells were treated with hUMSCs-CM in the presence of LPS $(200 \mathrm{ng} / \mathrm{ml})$. The supernatant was used for IL-1ra ELISA analysis $24 \mathrm{~h}$ later. Independent experiments were triplicated on different days.

\section{Knockdown of CREB}

After the raw 264.7 cells $\left(2 \times 10^{5}\right)$ were plated in 6-well plates, transfection with $100 \mathrm{nM}$ of siRNA for CREB (siCREB) and control (siCtr) was performed for $48 \mathrm{~h}$ using Lipofectamine 3000 reagent (Invitrogen, MA) according to the manufacturer's instructions. The siCREB (sense $5^{\prime}$ CCACAAAUCAGAUUAAUUUUU-3', antisense 5'AAAUUAAUCUGAUUUGUGGUU- ${ }^{\prime}$ ) and $s i C t r$ (sense 5'-ACGUGACACGUUCGGAGAA-3', antisense 5' UUCUCCGAACGUGUCACGU-3') were purchased (Genolution Pharmaceuticals, Inc., Seoul, Korea). After transfection, the RNA and proteins were isolated and used for PCR and western blot analysis, respectively.

\section{Enzyme-linked immunosorbent assay (ELISA)}

The IL-1 $\beta$ and IL-1ra levels were measured in the supernatants of raw 264.7 cells using commercially available ELISA kits (IL-1 $\beta$ Quantikine ELISA and IL-1ra Quantikine ELISA kits, R\&D Systems, MN). The MPO was measured in the supernatants of rat brain tissue using the MPO assay kit (Hycult Biotech, Uden, the Netherlands). The ELISA procedure was conducted according to the manufacturer's instructions. Independent experiments were duplicated on different days.

\section{Statistical analysis}

Statistical analyses were performed using Statistical Analysis System program (Enterprise 4.1; SAS Korea) and MedCalc statistical software (MedCalc software, ver. 11.6, Mariakerke, Belgium). The statistical significance between two groups in the histological or infarct size measurements was analyzed by the Mann-Whitney $U$ test. The statistical significance of multiple comparisons for realtime PCR or ELISA was analyzed using the Kruskal-Wallis test with a post hoc Conover's test for pairwise comparisons of subgroups. The analysis of functional tests was performed using the two-way mixed analysis of variance (mixed ANOVA) test. Statistical significance was considered at $p<0.05$ and $p<0.001$, and all values are presented as the means \pm standard error of the mean (SEM). The statistical analysis of the microarray data was described above.

\section{Results}

IV-hUMSCs in the acute phase of cerebral ischemia induces functional improvement and reduction of neuronal damage in MCAo rats

The study design for in vivo experiments is shown in Supplementary Figure S2. All the outcome measurements were conducted by observers who were blinded to the treatments. During the 8 weeks of the study period after 


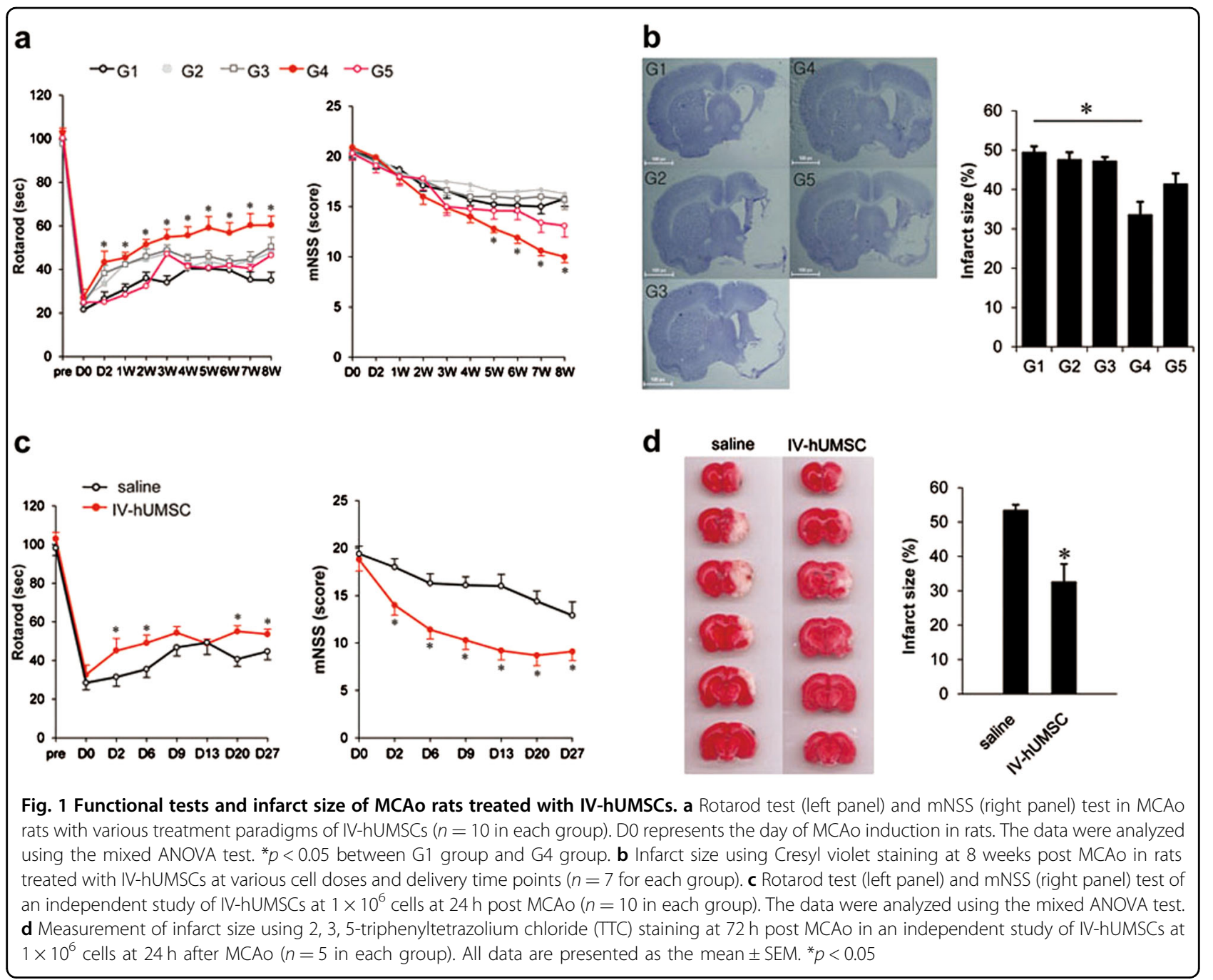

the induction of 90 min-middle cerebral artery occlusion (MCAo), there was no significant difference in mortality in each treatment group $(p>0.05)$. In the rotarod test, only the rats treated with hUMSCs at a dose of $1 \times 10^{6}$ cells at $24 \mathrm{~h}$ post MCAo (G4 group) showed a significant functional improvement, compared with the G1 group (saline treatment) (Fig. 1a). In the modified neurological severity score (mNSS) test, functional improvement was also observed in the G4 group (Fig. 1a). Interestingly, rats treated with the same dose of hUMSCs as in the G4 group, but treated in the delayed phase of ischemic stroke (G5 group), did not show any significant effects in the functional tests.

We also observed that the infarct size at 8 weeks post MCAo was significantly smaller in the G4 group than in the G1 group (G4 vs. G1: $33.6 \pm 3.3 \%$ vs. $49.4 \pm 1.5 \%$, $p=0.004$ ) (Fig. 1b). No difference in infarct size was observed in the other group when compared with the G1 group.
We next conducted an independent experiment to investigate the effects of IV-hUMSCs at a dose of $1 \times 10^{6}$ cells in the acute phase of cerebral ischemia in detail. Rats treated with $1 \times 10^{6}$ IV-hUMSCs at $24 \mathrm{~h}$ after MCAo (IV-hUMSC group) showed a significant improvement over 4 weeks of functional tests (Fig. 1c) and had a smaller infarct size at $72 \mathrm{~h}$ after MCAo induction, compared with the rats treated with saline alone (saline group) (IV-hUMSCs vs. saline: $31.9 .3 \pm 5.9 \%$ vs. $53.4 \pm 2.5 \%$, $p=0.005$ ) (Fig. 1d). We next investigated the effects of IV-hUMSCs on neuronal apoptosis using terminal deoxynucleotidyl transferase dUTP nick-end labeling (TUNEL) assay. In the saline group, there were numerous TUNEL-positive cells in the peri-infarct area at $72 \mathrm{~h}$ after MCAo, suggesting that extensive neuronal apoptosis occurred in the acute phase of cerebral ischemia. In the IV-hUMSC group, the number of TUNEL-positive cells in the peri-infarct area at $72 \mathrm{~h}$ post MCAo was lower than in the saline group (IV-hUMSCs vs. saline: $22.1 \pm 1.9 \%$ vs. 
$39.5 \pm 3.8 \%, p=0.006$ ) (Supplementary Figure S3). These results clearly supported our findings from the initial experiment, which revealed that IV-hUMSCs at a dose of $1 \times 10^{6}$ cells at $24 \mathrm{~h}$ post MCAo, can give rise to a significant functional improvement as well as a reduction of infarct size. Based on these results, we conducted detailed biochemical and histological analyses comparing rats treated with IV-hUMSCs at a dose of $1 \times 10^{6}$ cells at $24 \mathrm{~h}$ after MCAo (IV-hUMSC group) and rats treated with saline alone (the saline group) as a control.

\section{IV-hUMSCs in the acute phase of cerebral ischemia attenuates post-ischemic inflammation}

We first conducted an immunohistochemical analysis of post-ischemic inflammation between the IV-hUMSC group and the saline group. In the saline group, numerous ED-1 (the rat homolog of human CD68)-positive cells and ionized calcium-binding protein adapter molecule 1 (Iba-1)-positive cells were found in the peri-infarct area at $72 \mathrm{~h}$ post MCAo. In the IV-hUMSC group, the numbers of ED-1-positive cells (IV-hUMSCs vs. saline: $20.2 \pm 2.1 \%$ vs. $39.3 \pm 2.9 \%, p=0.006$ ) and Iba-1-positive cells (IVhUMSCs vs. saline: $25.6 \pm 2.1 \%$ vs. $34.9 \pm 2.9 \%, p=0.017$ ) in the peri-infarct area were reduced compared with the saline treatment (Fig. 2a). In addition, the proportion of inducible nitric oxide synthase (iNOS)-positive cells in ED-1-positive cells was lower (IV-hUMSCs vs. saline: $43.7 \pm 4.3 \%$ vs. $60.3 \pm 5.1 \%, p=0.003)$, whereas the proportion of CD206-positive cells in ED-1-positive cells (IVhUMSCs vs. saline: $66.5 \pm 3.3 \%$ vs. $34.1 \pm 4.3 \%, p<0.001$ ) was higher in the IV-hUMSC group than in the saline group (Fig. 2b).

We next evaluated the changes in neutrophil infiltration into the infarct area after IV-hUMSC treatment. According to the neutrophil elastase (ELANE) immunostaining, fewer ELANE-positive cells were observed in the IV-hUMSC group than in the saline group (IVhUMSCs vs. saline: $4.9 \pm 0.9 \%$ vs. $16.7 \pm 1.9 \%, p=0.001$ ) (Fig. 2c). The results from the myeloperoxidase (MPO) ELISA indicated that the level of MPO at $72 \mathrm{~h}$ post MCAo was lower in the IV-hUMSC group than in the saline group (IV-hUMSCs vs. saline: $74.1 \pm 4.8 \mathrm{pg} / \mathrm{ml}$ vs. $225.1 \pm$ $4.7 \mathrm{pg} / \mathrm{ml}, p<0.001$ ) (Fig. 2d).

Real-time polymerase chain reaction (PCR) analysis of inflammation-related genes further revealed that cerebral ischemia induced an upregulation of the IL1B (interleukin-1 $\beta$ coding gene), TNF (TNF- $\alpha$ coding gene), $M M P 9$ (matrix metalloproteinase 9 coding gene), IL6 (IL6 coding gene), IL10 (IL-10 coding gene), and TGFB1 (TGF- $\beta 1$ coding gene) genes (Fig. 3). However, in the IVhUMSC group, the expression of IL1B, TNF, and MMP9 was significantly downregulated compared with the saline group (Fig. 3). Expression levels of IL4 (IL-4 coding gene) in the IV-hUMSC group and the saline group were not different from the controls. These findings provide histological and biochemical evidence of the effect of IV administration of hUMSCs on post-ischemic inflammation in cerebral ischemia.

\section{IV-hUMSCs in the acute phase of cerebral ischemia increases the expression of endogenous IL-1ra in the ischemic brain}

To delineate the molecular mechanism of IV-hUMSCs in the acute phase of cerebral ischemia, we performed mRNA microarray of brain tissues from the IV-hUMSC group and the saline group at $72 \mathrm{~h}$ post MCAo. The brain tissues from rats without MCAo were regarded as a control (control group). Comparison of gene expression profiles between the control group and the saline group at $72 \mathrm{~h}$ post MCAo showed that a total of 595 transcripts (553 transcripts were upregulated and 42 transcripts were downregulated) were differentially expressed in the saline group (Supplementary Table S3). When gene expression profiles were compared between the IV-hUMSC group and the saline group, a total of 85 transcripts (77 transcripts were upregulated and 8 transcripts were downregulated) were differentially expressed in the IV-hUMSC group (Supplementary Table S4). Among them, IL1RN, a gene encoding the interleukin-1 receptor antagonist (IL1ra), was one of the most strongly upregulated proteincoding genes in the IV-hUMSC group compared with the saline group (Fig. 4a). IL-1 ra is a natural antagonist of IL-1 and plays a role in the regulation of IL-1-mediated inflammation in cerebral ischemia ${ }^{14,15}$. Thus, we hypothesized that the therapeutic effect of IV-hUMSCs on cerebral ischemia is mediated by IL-1ra and further investigated the change in IL-1ra expression in ischemic brain after IV-hUMSC treatment. Real-time PCR analysis indicated that the expression of ILIRN was upregulated in cerebral ischemia and was further upregulated in IVhUMSCs after cerebral ischemia (Fig. 4b). In addition, the level of IL-1ra protein in the ischemic brain tissue at $72 \mathrm{~h}$ post MCAo was also increased in the IV-hUMSC group compared with the saline group (IV-hUMSCs vs. saline: $237.5 \pm 49.0 \mathrm{pg} / \mathrm{ml} \quad$ vs. $\quad 119.6 \pm 15.6 \mathrm{pg} / \mathrm{ml}, \quad p=0.01)$ (Fig. 4c).

To identify the cell subpopulation that contributes to IL-1ra upregulation, we performed a double immunochemical study staining using antibodies against IL-1ra and ED-1 (microglial markers), NeuN (a neuronal marker), or Reca-1 (an endothelial marker) in ischemic brains. We observed that the proportion of IL-1ra-positive cells in ED-1-positive cells (IV-hUMSCs vs. saline: $34.8 \pm 2.5 \%$ vs. $22.1 \pm 3.4 \%, p=0.01$ ) was higher in the IV-hUMSC group than in the saline group at $72 \mathrm{~h}$ post MCAo (Fig. 4d). By contrast, the proportion of IL-1ra-positive cells in either Reca-1-positive cells or NeuN-positive cells was relatively low (less than 7\%) and was not different 


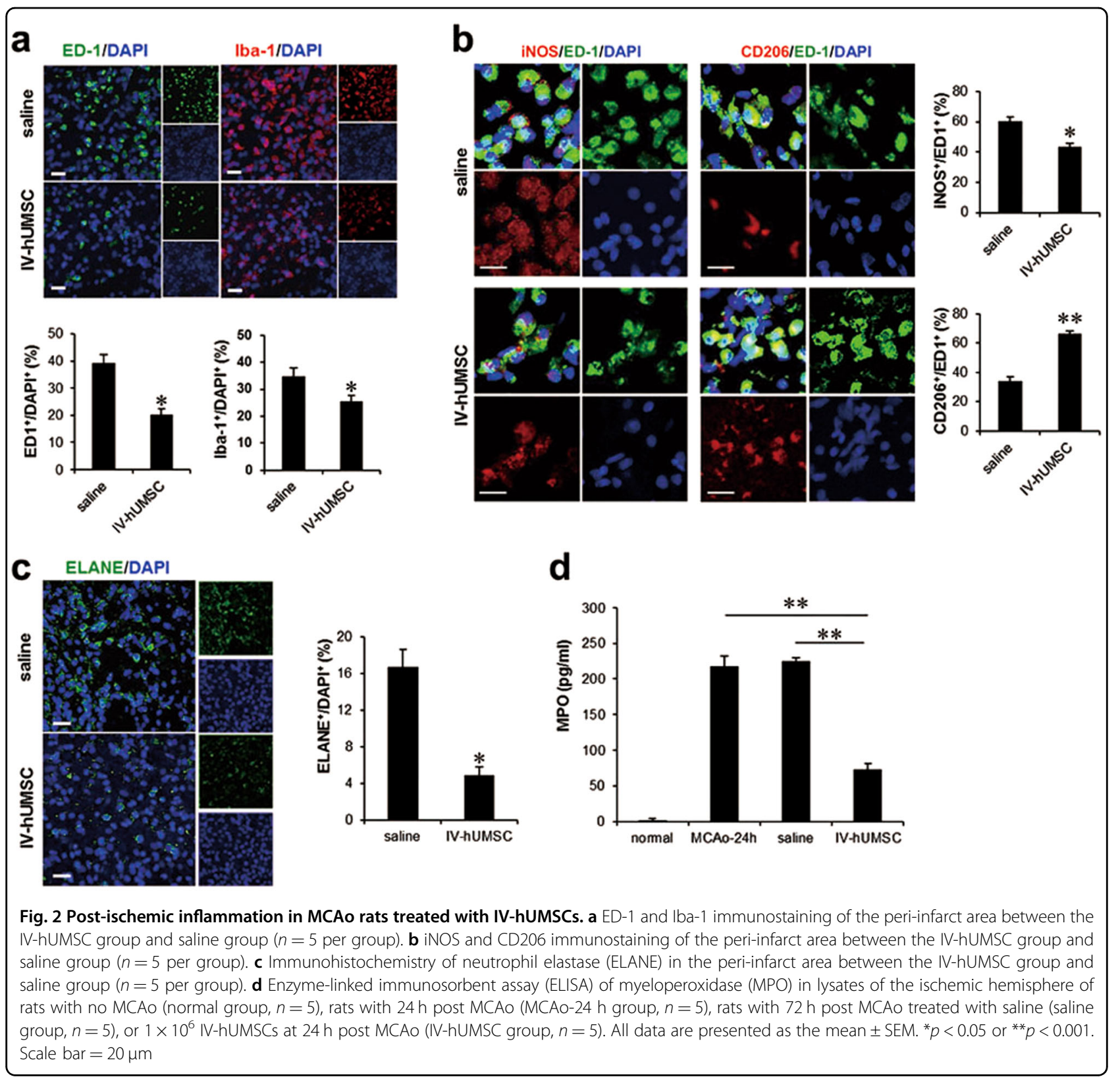

between the IV-hUMSC group and the saline group. It is known that although MSC secretes IL-1ra, the proportion of IL-1ra-secreting cells is very low in human MSCs (less than $5 \%$ of MSCs) ${ }^{16}$. We observed that IV administered hUMSCs were hardly detectable in the ischemic brain (less than $1 \%$ of administered cells) at $72 \mathrm{~h}$ and 4 weeks post MCAo by immunostaining using a human-specific nuclear antibody. In the ELISA experiment, IL-1ra was not detected in the conditioned medium of hUMSCs (hUMSCs-CM) $(<3.2 \mathrm{pg} / \mathrm{ml})$. This finding suggests that the increase in IL-1ra originated from inflammatory cells in the ischemic brain, such as microglia and macrophages, rather than from the transplanted cells.
hUMSCs upregulate IL-1ra release by activation of CREB in macrophages

In addition to microglia in the central nervous system, circulating macrophages also play a role in post-ischemic inflammation by infiltrating into brain parenchyma and releasing pro-inflammatory cytokines, including $\mathrm{IL}-1^{13}$. It is known that MSCs polarize circulating macrophages into anti-inflammatory phenotype and recruit a number of inflammatory cells that contribute to healing of the damaged tissue ${ }^{17}$. Thus, we investigated the effect of hUMSCs-CM on IL-1ra expression in a mouse macrophage cell line (raw 264.7 cells). Treatment with lipopolysaccharide (LPS) strongly elevated the levels of both IL- 


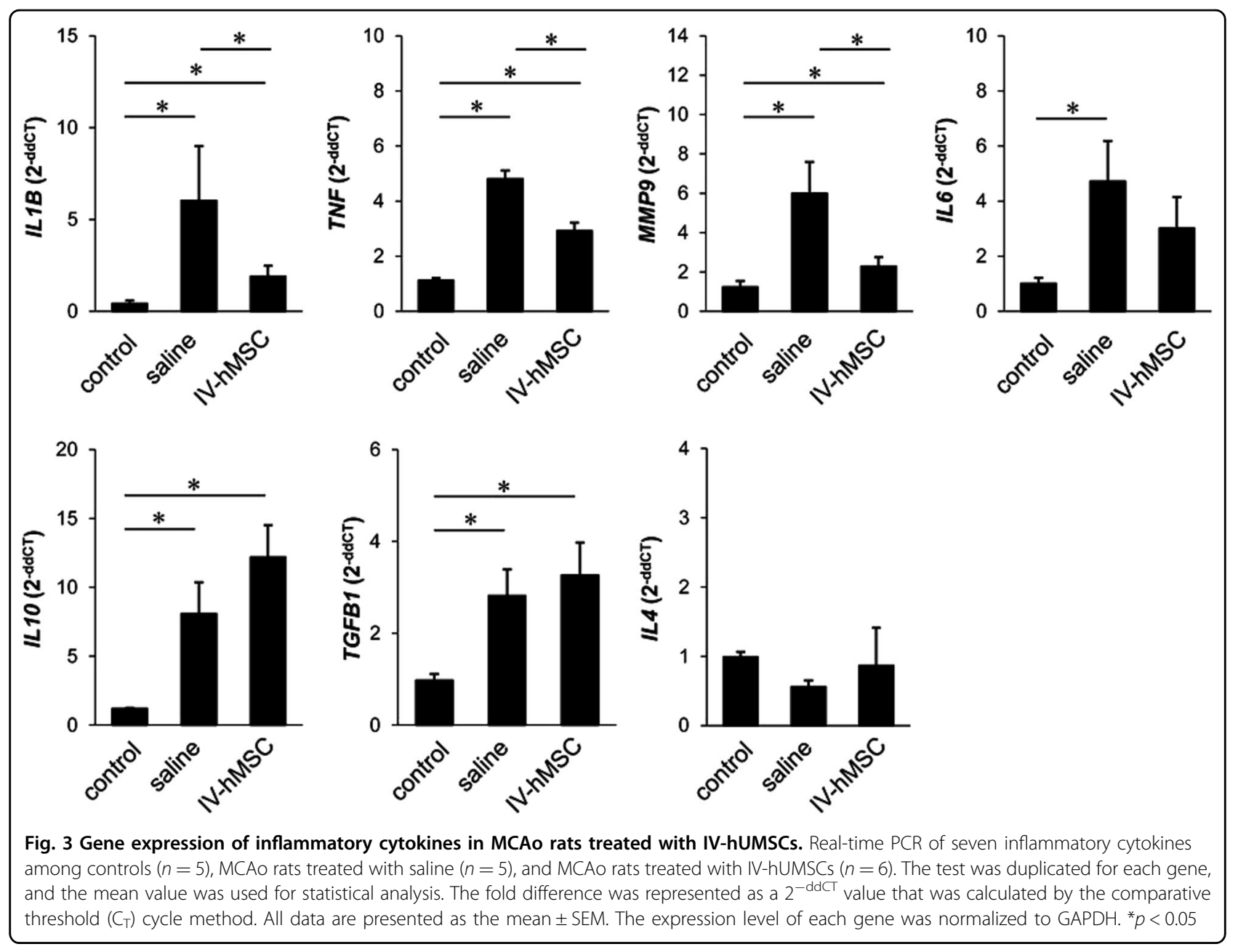

$1 \beta$ and IL-1ra in raw 264.7 cells (Fig. 5a). IL-1ra expression was upregulated by NF- $\mathrm{BB}$ signaling in response to the inflammatory milieu, such as IL-1 $\beta$ activation, as a compensatory mechanism ${ }^{18}$. When raw 264.7 cells were co-treated with LPS and hUMSCs-CM, the IL-1 $\beta$ level was reduced, whereas the IL-1ra level was further increased (Fig. 5a). This result suggests that there could be some mechanism(s) other than NF- $\mathrm{kB}$ that plays a role in the increase in IL-1ra after treatment with hUMSCs-CM. We further investigated the role of cAMP-response element-binding protein (CREB) in hUMSCs-mediated IL-1ra expression in macrophages. Western blot analysis showed that expression of phosphorylated CREB (p-CREB) proteins was significantly increased but phosphorylated NF- $\mathrm{kB}$ (p-NF- $\mathrm{kB}$ ) was decreased in raw 264.7 cells when co-treated with hUMSCs-CM and LPS, compared to those treated with LPS alone (Fig. 5b). Immunocytochemistry of p-CREB and p-NF- $k B$ showed that co-treatment with LPS and hUMSCs-CM strongly increased the expression of p-CREB in raw 264.7 cells compared with the treatment with LPS alone
(Supplementary Figure S4). When hUMSCs-CM was cotreated with CREB inhibitor (KG501), the effect of hUMSCs-CM on the IL-1ra increase was reduced in a dose-dependent manner (Fig. 5c).

Furthermore, transfection with siRNA for CREB (siCREB) also reduced the expression of IL-1ra protein in raw 264.7 cells (Fig. 5d). In the LPS-stimulated raw 264.7 cells, knockdown of CREB reduced the enhanced expression of ILIRN, which could also be induced by treatment with hUMSCs-CM (Supplementary Figure S5). These results strongly suggest that hUMSCs can give rise to the release of IL-1ra through the activation of CREB in macrophages via a paracrine mechanism.

\section{Discussion}

Through comprehensive functional, histological, and biochemical analyses, we provide important preclinical evidence for the therapeutic efficacy of IV administration of hUMSCs in a rodent model of stroke. Previous studies have already shown that IV administration of human MSCs leads to positive results for treating stroke both in 

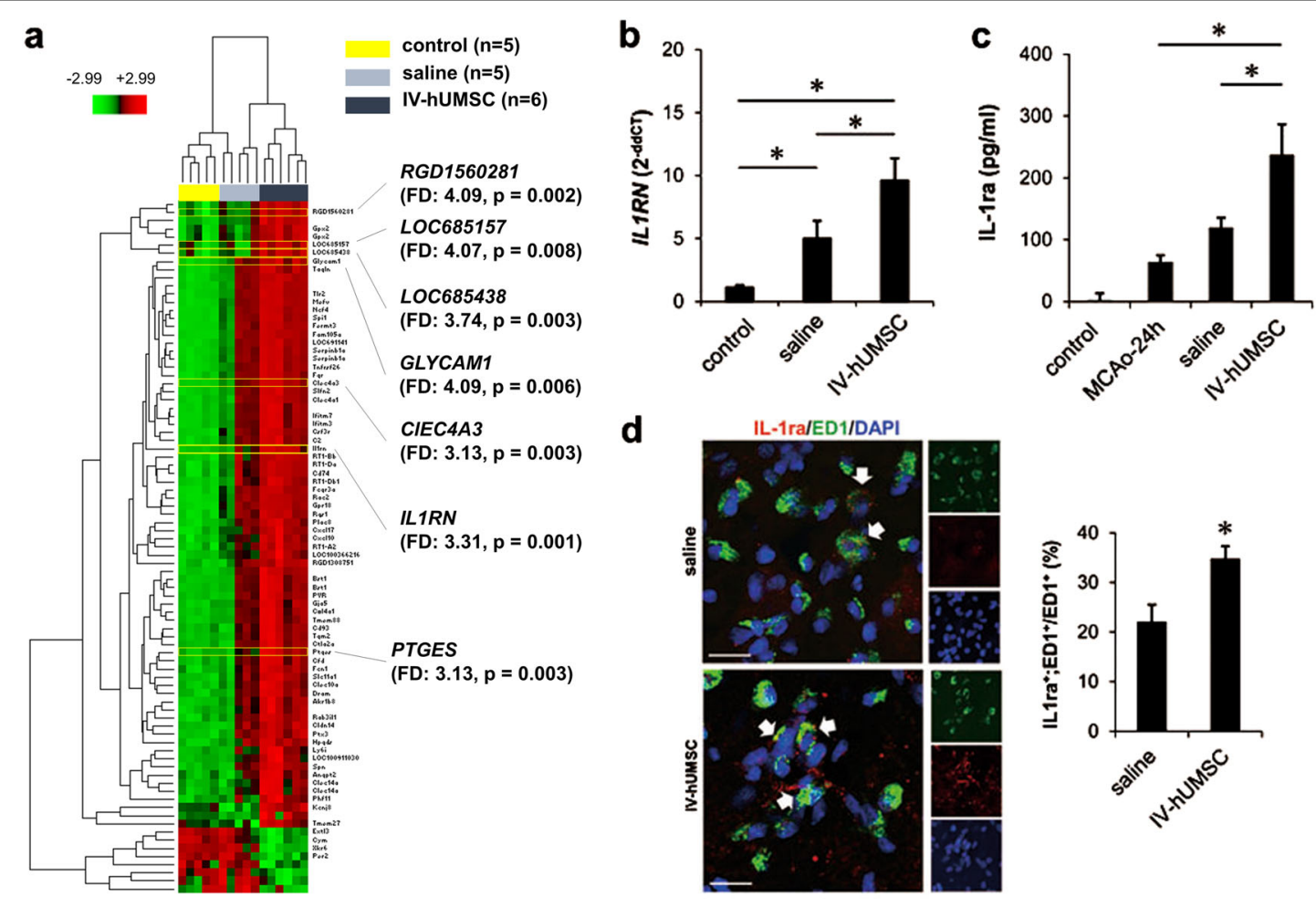

Fig. 4 IL-1 ra expression in MCAo rats treated with IV-hUMSCs. a mRNA microarray analysis on the ischemic brain of MCAo rats treated with IVhUMSCs $(n=6)$ or saline $(n=5)$ at $24 \mathrm{~h}$ post MCAo. Normal rat brain was used as a control $(n=5)$. A heat map of a total of 85 differentially expressed genes (fold difference $\geq 2$ and $p<0.01$ after Benjamini-Hochberg's correction) is presented in the left panel. The transcripts listed in the right panel represent the most highly expressed transcripts between the IV-hUMSC group and saline group (fold difference $\geq 3$ and $p<0.01$ after Benjamini-Hochberg's correction). FD represents the fold difference. $p$ represents the $p$ value. $\mathbf{b}$ Real-time PCR of ILIRN in brain tissue in the IVhUMSC group and saline group. The fold difference was represented as a $2^{- \text {ddCT }}$ value. ${ }^{*} p<0.05$. c Enzyme-linked immunosorbent assay (ELISA) of IL$1 \mathrm{ra}$ in the ischemic hemisphere of rats with no MCAo (control), rats with $24 \mathrm{~h}$ post MCAo (MCAo- $24 \mathrm{~h}$ ), rats with $72 \mathrm{~h}$ post MCAo treated with saline (saline group), and rats with $72 \mathrm{~h}$ post MCAo treated with $1 \times 10^{6} \mathrm{IV}$-hUMSCs at $24 \mathrm{~h}$ post MCAo (IV-hUMSC group) ( $n=5 \mathrm{in}$ each group). $\mathbf{d}$ IL-1 ra and ED-1 double immunostaining (arrows) in the ischemic brain in the IV-hUMSC group and saline group ( $n=5$ in each group). All data are presented as the mean \pm SEM. ${ }^{*} p<0.05$. Scale bar $=20 \mu \mathrm{m}$

animal $^{6,19-21}$ and human studies ${ }^{1,2,22}$. In the present study, we found that IV administration of at least $1 \times 10^{6}$ MSCs during the acute phase of cerebral ischemia is required to induce functional and histological improvement in a rat MCAo model. This finding is consistent with previous studies, in which the effective dose of IVMSCs generally ranges from $5 \times 10^{5}$ to $3 \times 10^{6}$ in the rat stroke model $^{23}$. As shown in Fig. 1, we conducted two independent experiments, in which hUMSCs were administered to rats $24 \mathrm{~h}$ post MCAo. Although significant behavioral recovery was equally observed in each experiment, we also observed subtle differences in recovery patterns, which may be attributable to the variations in animals and their infarct conditions. There is also a possibility that this difference was due to the scoring of each behavioral test by independent researchers at different time points. Nevertheless, both behavioral scores support the occurrence of behavioral improvements following IV-hUMSCs at $24 \mathrm{~h}$ post MCAo.
We found that single treatment of IV-hUMSCs in the sub-acute stage was not effective, which was supported by previous studies showing that acute treatment with stem cells showed better results than chronic treatment ${ }^{24-27}$. In ischemic stroke, the long-term outcome is strongly dependent on the severity of functional deficits from the acute phase of stroke, during which neuroinflammation and neuronal death extensively develop ${ }^{13}$. Therefore, we strongly suggest that treatment with IV-hUMSCs should be performed in the acute phase of stroke to achieve the best therapeutic effects in the clinical setting.

Our results showed that the main therapeutic mechanism of IV-hUMSCs in the acute phase of cerebral ischemia is the modulation of post-ischemic inflammation, especially through the activation of endogenous IL$1 \mathrm{ra}$ in the ischemic brain. Immunohistochemical analyses revealed that most cells expressing IL-1ra co-stained with ED-1, a marker for microglia and peripheral blood-borne macrophages. When IV-hUMSCs were treated, IL-1ra 


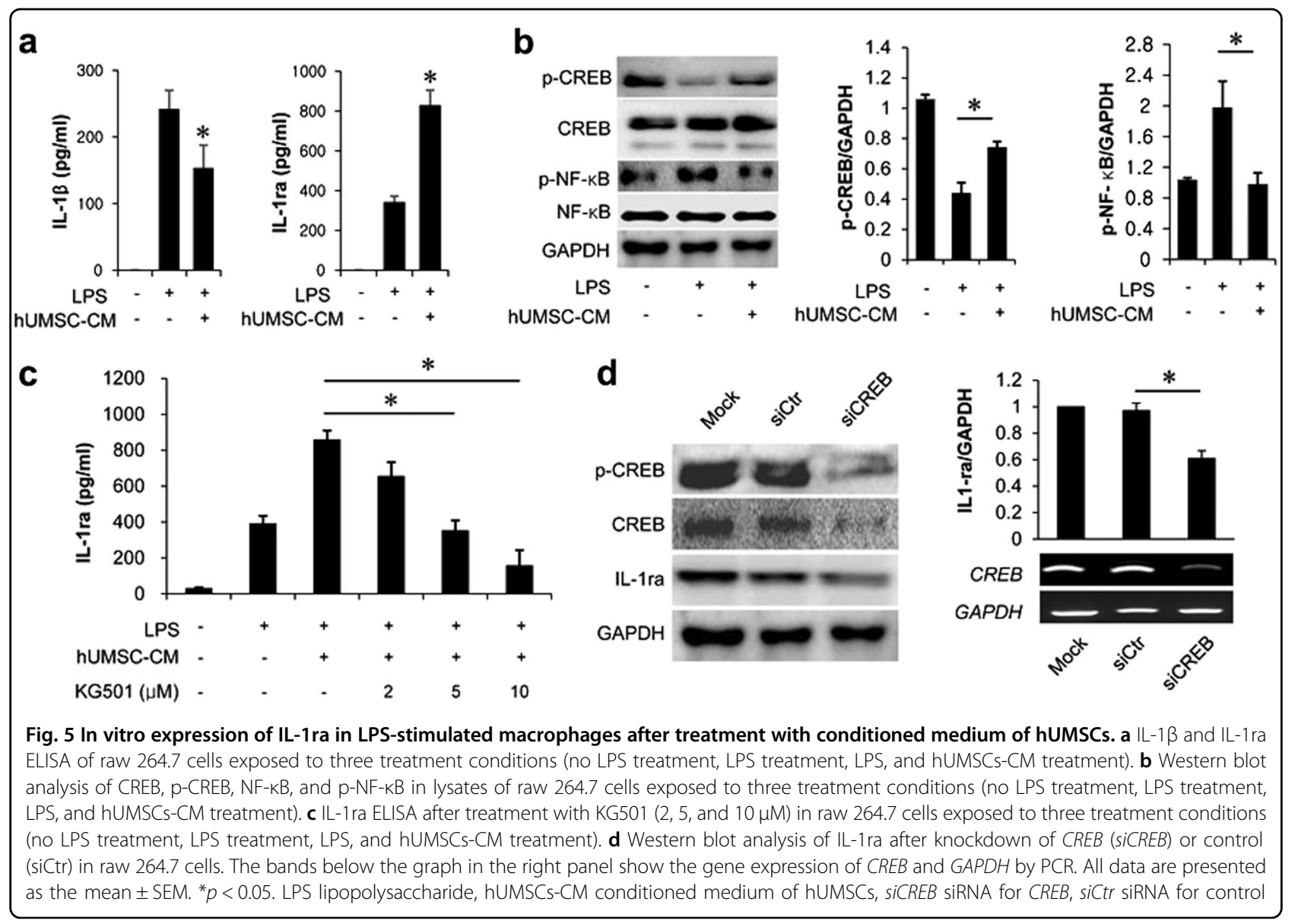

mRNA expression was highly upregulated in the ischemic brain by whole gene expression analysis. We also observed that the IL-1ra protein level was increased under the same conditions. IL-1ra is one of the key molecules known to regulate post-ischemic inflammation, and it is rapidly upregulated in the brain in response to hypoxia or inflammation ${ }^{14,18,28}$. Microglia and circulating macrophages can release anti-inflammatory cytokines as well as pro-inflammatory cytokines in response to stress or brain injury ${ }^{29,30}$. A previous in vivo study has demonstrated the central role of IL-1ra in stroke animal models as follows: IL-1ra knockout mice showed a larger infarct size following cerebral ischemia, whereas peripherally administered bone marrow cells in IL-1ra transgenic mice were neuroprotective against cerebral ischemia by increasing the release of IL-1ra in microglia and circulating macrophages $^{31}$. Treatment with recombinant IL-1ra improved functional impairment in an animal stroke model ${ }^{32}$ and ameliorated the clinical course of stroke ${ }^{33}$, indicating its beneficial effect against cerebral ischemia. In cerebral ischemia, inflammatory responses originating from the resident microglia and blood-borne macrophages peak at 24-72 $\mathrm{h}$ after cerebral ischemia and may persist for several weeks after the initial injury ${ }^{34}$. It is well known that there is a prominent shift in inflammatory responses during the early stage of ischemic stroke ${ }^{35}$.

It has been reported that there is a prominent shift of M2 to M1 phenotypes from 1 day up to 14 days in a rodent model of transient cerebral ischemia ${ }^{35}$, suggesting the pathological contribution of M1 polarization to postischemic inflammation during this period. For this reason, we examined the responses of the ischemic brain following the IV administration of hUMSCs at $72 \mathrm{~h}$ after MCAo.

Our results indicated that, at $72 \mathrm{~h}$ post MCAo, treatment with hUMSCs at $24 \mathrm{~h}$ post MCAo significantly increased the proportion of anti-inflammatory M2-like cells (CD206positive cells) compared with pro-inflammatory M1-like cells (iNOS-positive cells) in the peri-infarct area after cerebral ischemia, suggesting a polarization of M1 to M2 phenotypes within $48 \mathrm{~h}$ after IV-hUMSC treatment. M2-like microglia and/or macrophages are known to produce several anti-inflammatory cytokines, including IL-1 $\mathrm{ra}^{36}$. We hypothesize that IV-hUMSCs probably induce the polarization of CNS immune cells toward the anti-inflammatory phenotype, which subsequently increases the release of IL-1ra.

In our study, we further showed that CREB is required for hUMSC-mediated IL-1ra release in macrophages. CREB plays a role in neuronal survival and plasticity in the 
nervous system. A recent study has demonstrated the role of CREB as an important regulator of inflammation by showing that activation of CREB is required for the upregulation of anti-inflammatory genes in macrophages $^{37}$. Interestingly, p-CREB binds to one CRE promoter site, located between nucleotides 93 and 113 upstream of the ILIRN open reading frame, suggesting a possible direct biological link between CREB and IL-1 $\mathrm{ra}^{38}$. It has also been shown that treatment with human adipose-derived MSCs activates CREB, which subsequently prevent the progression of phenotypes in a Huntington's disease model ${ }^{39}$. Taken together, these findings provide strong evidence for the role of CREB in the hUMSC-mediated upregulation of IL-1ra in macrophages. However, it is still largely unknown how CREB is regulated in the central nervous system in response to cerebral ischemia, possibly in combination with IVhUMSCs in vivo. To better develop MSC-based stroke therapies, it will be important to elucidate the mechanisms underlying the interplay between CREB and microglial IL-1ra in response to IV-hUMSCs.

In conclusion, we showed that treatment with IVhUMSCs in acute cerebral ischemia can give rise to functional improvements and reduced neuronal damage in a rodent model of stroke. In addition, we demonstrated that activation of endogenous IL-1ra is one of the therapeutic mechanisms of IV-hUMSCs in cerebral ischemia. Our results provide a scientific basis for the rationale of the treatment with IV-hUMSCs for clinical applications in human stroke. In contrast to autologous MSCs, allogeneic hUMSCs are readily available for the treatment of acutephase stroke. Therefore, our GMP-manufactured hUMSCs could serve as a good candidate for an "off-the-shelf" cell source for the treatment of patients with acute stroke.

\section{Acknowledgements}

This study was supported by grants from the Korea Healthcare Technology R\&D project through the Korea Health Industry Development Institute (KHIDI), funded by the Ministry for Health, Welfare \& Family Affairs, Republic of Korea (H116C1559 and HI14C3297), and a grant of Basic Science Research Program through National Research Foundation of Korea (NRF) funded by the Ministry of Education (NRF-2015R1D1A1A01060263).

\section{Author details \\ 'Department of Neurology, CHA Bundang Medical Center, CHA University, Seongnam, Republic of Korea. ${ }^{2}$ Department of Biomedical Science, CHA University, Seongnam, Republic of Korea. ${ }^{3}$ Development Division, CHA Biotech, Co. Ltd., Seongnam, Republic of Korea. ${ }^{4}$ Department of Biotechnology, CHA University, Seongnam, Republic of Korea. ${ }^{5} \mathrm{CHA}$ Stem Cell Institute, CHA University, Seongnam, Republic of Korea}

\section{Author contributions}

S.-H.O., C.C., J.-E.N., O.-J.K., and J.S. conceived and designed the experiments. S.H.O., C.C., and J.-E.N. conducted the animal experiments. J.M.S., J.H.K., and H.-J. K. prepared the mesenchymal stromal cells. S.-H.O., C.C., and J.-M.L. conducted the immunoassays. N.L., Y.-W.J., I.J., and H.-S.K. performed the data analysis. S.-H. O., C.C., J.-E.N., O.-J.K., and J.S. wrote the manuscript. All authors have read and approved the submission of the manuscript.
Conflict of interest

The authors declare that they have no conflict of interest.

\section{Publisher's note}

Springer Nature remains neutral with regard to jurisdictional claims in published maps and institutional affiliations.

Supplementary information accompanies this paper at https://doi.org/ 10.1038/s12276-018-0041-1.

Received: 5 October 2017 Accepted: 21 December 2017.

Published online: 13 April 2018

\section{References}

1. Bang, O. Y., Lee, J. S., Lee, P. H. \& Lee, G. Autologous mesenchymal stem cell transplantation in stroke patients. Ann. Neurol. 57, 874-882 (2005).

2. Lee, J. S. et al. A long-term follow-up study of intravenous autologous mesenchymal stem cell transplantation in patients with ischemic stroke. Stem Cells 28, 1099-1106 (2010).

3. Ranganath, S. H., Levy, O., Inamdar, M. S. \& Karp, J. M. Harnessing the mesenchymal stem cell secretome for the treatment of cardiovascular disease. Cell Stem Cell 10, 244-258 (2012)

4. Weiss, M. L. et al. Immune properties of human umbilical cord Wharton's jellyderived cells. Stem Cells 26, 2865-2874 (2008).

5. Koh, S. H. et al. Implantation of human umbilical cord-derived mesenchymal stem cells as a neuroprotective therapy for ischemic stroke in rats. Brain Res. 1229, 233-248 (2008).

6. Zhang, L. et al. Delayed administration of human umbilical tissue-derived cells improved neurological functional recovery in a rodent model of focal ischemia. Stroke 42, 1437-1444 (2011).

7. Kim, S. M. et al. Alternative xeno-free biomaterials derived from human umbilical cord for the self-renewal ex-vivo expansion of mesenchymal stem cells. Stem Cells Dev. 22, 3025-3038 (2013).

8. Bai, L. et al. Hepatocyte growth factor mediates mesenchymal stem cellinduced recovery in multiple sclerosis models. Nat. Neurosci. 15, 862-870 (2012).

9. Longa, E. Z., Weinstein, P. R., Carlson, S. \& Cummins, R. Reversible middle cerebral artery occlusion without craniectomy in rats. Stroke 20, 84-91 (1989).

10. Chang, D. J. et al. Therapeutic potential of human induced pluripotent stem cells in experimental stroke. Cell Transplant. 22, 1427-1440 (2013).

11. Oh, S. H. et al. Early neuroprotective effect with lack of long-term cell replacement effect on experimental stroke after intra-arterial transplantation of adipose-derived mesenchymal stromal cells. Cytotherapy 17, 1090-1103 (2015).

12. Choi, C. et al. Attenuation of post-ischemic genomic alteration by mesenchymal stem cells: a microarray study. Mol. Cells 39, 337-344 (2016).

13. ladecola, C. \& Anrather, J. The immunology of stroke: from mechanisms to translation. Nat. Med. 17, 796-808 (2011).

14. Boutin, H. et al. Role of IL-1alpha and IL-1beta in ischemic brain damage. J. Neurosci. 21, 5528-5534 (2001)

15. Wang, X., Barone, F. C., Aiyar, N. V. \& Feuerstein, G. Z. Interleukin-1 receptor and receptor antagonist gene expression after focal stroke in rats. Stroke $\mathbf{2 8}$, 155-161 (1997).

16. Ortiz, L. A. et al. Interleukin 1 receptor antagonist mediates the antiinflammatory and antifibrotic effect of mesenchymal stem cells during lung injury. Proc. Natl Acad. Sci. USA 104, 11002-11007 (2007).

17. Nemeth, K. et al. Bone marrow stromal cells attenuate sepsis via prostaglandin $E(2)$-dependent reprogramming of host macrophages to increase their interleukin-10 production. Nat. Med. 15, 42-49 (2009).

18. Gabay, C., Smith, M. F., Eidlen, D. \& Arend, W. P. Interleukin 1 receptor antagonist (IL-1Ra) is an acute-phase protein. J. Clin. Invest. 99, 2930-2940 (1997).

19. Kim, J. M. et al. Systemic transplantation of human adipose stem cells attenuated cerebral inflammation and degeneration in a hemorrhagic stroke model. Brain Res. 1183, 43-50 (2007). 
20. Ikegame, Y. et al. Comparison of mesenchymal stem cells from adipose tissue and bone marrow for ischemic stroke therapy. Cytotherapy 13, 675-685 (2011).

21. Deng, Y. B. et al. Intravenously administered BMSCs reduce neuronal apoptosis and promote neuronal proliferation through the release of VEGF after stroke in rats. Neurol. Res. 32, 148-156 (2010).

22. Honmou, O. et al. Intravenous administration of auto serum-expanded autologous mesenchymal stem cells in stroke. Brain 134, 1790-1807 (2011).

23. Liu, $X$. et al. Cell based therapies for ischemic stroke: from basic science to bedside. Prog. Neurobiol. 115, 92-115 (2014).

24. Bliss, T. M., Andres, R. H. \& Steinberg, G. K. Optimizing the success of cell transplantation therapy for stroke. Neurobiol. Dis. 37, 275-283 (2010).

25. Guzman, R. et al. Intravascular cell replacement therapy for stroke. Neurosurg. Focus 24, E15 (2008)

26. lihoshi, S., Honmou, O., Houkin, K., Hashi, K. \& Kocsis, J. D. A therapeutic window for intravenous administration of autologous bone marrow after cerebral ischemia in adult rats. Brain Res. 1007, 1-9 (2004).

27. Yang, B. et al. Therapeutic time window and dose response of autologous bone marrow mononuclear cells for ischemic stroke. J. Neurosci. Res. 89 833-839 (2011)

28. Glatz, T. et al. Peroxisome-proliferator-activated receptors gamma and peroxisome-proliferator-activated receptors beta/delta and the regulation of interleukin 1 receptor antagonist expression by pioglitazone in ischaemic brain. J. Hypertens. 28, 1488-1497 (2010).

29. Pinteaux, E., Rothwell, N. J. \& Boutin, H. Neuroprotective actions of endogenous interleukin-1 receptor antagonist (IL-1 ra) are mediated by glia. Glia $\mathbf{5 3}$ 551-556 (2006).
30. Rosito, M. et al. Trasmembrane chemokines CX3CL1 and CXCL16 drive interplay between neurons, microglia and astrocytes to counteract pMCAO and excitotoxic neuronal death. Front. Cell Neurosci. 8, 193 (2014).

31. Clausen, B. H. et al. Cell therapy centered on IL-1Ra is neuroprotective in experimental stroke. Acta Neuropathol. 131, 775-791 (2016).

32. Pradillo, J. M. et al. Reparative effects of interleukin-1 receptor antagonist in young and aged/co-morbid rodents after cerebral ischemia. Brain Behav. Immun. 61, 117-126 (2017).

33. Emsley, H. C. et al. A randomised phase II study of interleukin-1 receptor antagonist in acute stroke patients. J. Neurol. Neurosurg. Psychiatry 76, 1366-1372 (2005).

34. Jin, R., Yang, G. \& Li, G. Inflammatory mechanisms in ischemic stroke: role of inflammatory cells. J. Leukoc. Biol. 87, 779-789 (2010).

35. Hu, X. et al. Microglia/macrophage polarization dynamics reveal nove mechanism of injury expansion after focal cerebral ischemia. Stroke $\mathbf{4 3}$, 3063-3070 (2012)

36. Franco, R. \& Fernandez-Suarez, D. Alternatively activated microglia and macrophages in the central nervous system. Prog. Neurobiol. 131, 65-86 (2015).

37. Ruffell, D. et al. A CREB-C/EBPbeta cascade induces M2 macrophage-specific gene expression and promotes muscle injury repair. Proc. Natl Acad. Sci. USA 106, 17475-17480 (2009).

38. Corbett, G. T., Roy, A. \& Pahan, K. Gemfibrozil, a lipid-lowering drug, upregulates IL-1 receptor antagonist in mouse cortical neurons: implications for neuronal self-defense. J. Immunol. 189, 1002-1013 (2012).

39. Im, W. et al. Extracts of adipose derived stem cells slows progression in the R6/ 2 model of Huntington's disease. PLOS ONE 8, e59438 (2013). 